\title{
Effect of Sulphur Levels and Spray with some Natural Stimulant Substances on Productivity and Storability of Garlic Hala A. El-Sayed ${ }^{1}$; A. H. A. El-Morsy ${ }^{2}$ and Amira A. A. M. Kalifa ${ }^{2}$ \\ ${ }^{1}$ Vegetables \& Floriculture Department, Faculty of Agriculture, Mansoura University, Egypt. \\ ${ }^{2}$ Vegetables Research Department, Horticulture Research Institute, Agricultural Research Center, Giza, Egypt.
}

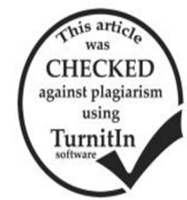

\begin{abstract}
Two field trials were conducted on garlic clone Sids-40, in the vegetable private Farm at Kafr Meet Faris, Dakahlia Governorate, during 2015/2016 and 2016/2017 seasons to study the effect of sulphur fertilizer levels (150,300 and $450 \mathrm{~kg}$ S/fed ) and spraying with some natural stimulants (yeast, moringa and chitosan ) on plant growth, yield and its components, as well as chemical constituents and storability of bulbs. In general, plants fertilized with sulphur elemental were better than unfertilized plants. Increasing of applied sulphur level from 150 to $450 \mathrm{~kg} \mathrm{~S} /$ fed significantly improved all studied growth characteristics, yield and its components in addition to chemical constituents, bulbing ratio and number of cloves/bulb which had a better values in both seasons. Moreover, sulphur application at 450 $\mathrm{kg} /$ fed significantly increased concentrations of N, P, K, S, TSS \% and volatile oils in cloves and enhanced storability. Foliar spray garlic plants 3 times past 45,60 and 75 days from planting with chitosan $(200 \mathrm{mg} / \mathrm{L})$ exceeded other foliar spray with some natural stimulants and formed the highest means of whole studied growth characteristics, yield and its components, chemical constituents and enhanced bulbing ratio, number of cloves/bulb storability in both seasons. The best results were obtained by application of $450 \mathrm{~kg} \mathrm{~S} / \mathrm{fed}$ and foliar spray with chitosan. This treatment achieved reduces weight loss at the end of the storage period reached to $20.3 \%$ and $20.5 \%$ in the first and the second seasons respectively comparing with the untreated ones. Therefore, this treatment could be recommended for raising garlic yield and improving bulb quality during the storage period under similar conditions to this work.
\end{abstract}

\section{INTRODUCTION}

Garlic (Allium sativumL.) is one of the most important bulb vegetable crops and is next to onion in importance. Increasing garlic yield and improving bulb quality depends on many factors especially that influence the plant growth throughout the growth period.

Sulphur (S) is an essential plant nutrient and its role in balanced fertilization and consequently in crop production is being increasingly appreciated. It is necessary for synthesis of amino acids like cystine, cystiene and methionine a component of vitamin A and activates certain enzyme systems in plants (Marschner, 2012). Application of sulphur element has an important role in soil $\mathrm{pH}$ decline that improved accessibility of some nutrient elements such as $\mathrm{P}, \mathrm{Fe}, \mathrm{Zn}, \mathrm{Mn}$ and $\mathrm{Cu}$ which were reflected on plant uptake and growth (Dahdouh et al., 1993; Mehana, 1994 and Mehana and Farag, 2000). Several investigators reported that garlic plants growth, yield and its quality as well as $\mathrm{N}, \mathrm{P}$ and $\mathrm{K}$ content in the plant tissues and bulbs were affected by sulphur application rate. In this respect, El-Morsy (2005), Jaggi and Raina (2005), Farooqui et al. (2009), Hore et al. (2014), Assefa et al. (2015), Youssif et al. (2015), Babaleshwar (2017), Patidar et al. (2017) and Shete et al. (2018) found that plant growth, total yield and quality were increased significantly with increasing level of applied sulphur.

Foliar application of natural stimulant substances is a widely used practice to correct nutritional deficiencies in plants caused by improper supply of nutrients to roots. In this regard, El-Morsy et al. (2011) found that foliar application garlic plants with yeast extract at $50 \mathrm{ml} / \mathrm{L}$ gave rise to significant increases in total yield, bulb weight and diameter and clove weight compared with the other treatments. Fawzy et al. (2012) showed that spraying of Chinese garlic plants with bio-stimulants among them chitosan had a significant effect on yield and chemical constitutes. They concluded that spraying of chitosan at rate of $3 \mathrm{~cm} / \mathrm{L}$ let to obtain the highest values of total yield per feddan as tons and average bulb diameter of Chinese garlic plants. Shalaby and El-Ramady (2014) indicated that foliar application with yeast showed the heaviest bulb weight and highest total bulb yield. Ahmed (2015) revealed that foliar application with dry yeast $(3$ or $4 \mathrm{~g} / \mathrm{L}$ ) or chitosan at 4 or $6 \mathrm{ml} / \mathrm{L}$ at $30,45,60$ and 75 days from planting date effectively increased bulb yield and its components in both seasons. Hegazi et al. (2016) showed that spraying with moringa leaves extract $10 \mathrm{~g} / \mathrm{L}$ increased average cured bulb diameter and weight, curing yield, blubs dry matter $\%$ and chemical constitutes as compared with the control treatment in both seasons.

Thus, this study was planned to decide the effects of sulphur levels and spraying with some natural stimulant on growth, yield and its components as well as chemical constituents and storability of garlic (Sids-40 cultivar) under the environmental conditions of Dakahlia Governorate, Egypt.

\section{MATERIALS AND METHODS}

Two field experiments were carried out in vegetable private Farm at Kafr Meet Faris, Dakahlia Governorate, during two growing seasons of 2015/2016 and 2016/2017, to study the effects sulphur application levels and foliar spray with some natural stimulant on garlic CV Sids-40 growth, yield and its components, as well as chemical constituents in leaves and cloves and bulb storability. This experiment included 16 treatments, which were arranged in split- plot design as follows:

a- Sulphur levels:

Control. ( Untreated ).

$150 \mathrm{~kg} \mathrm{~S} / \mathrm{fed}$

$300 \mathrm{~kg} \mathrm{~S} /$ fed.

$450 \mathrm{~kg} \mathrm{~S} / \mathrm{fed}$.

b- Natural stimulant substances treatments:

Unsprayed (tap water).

Foliar spray with yeast extract at the rate of $100 \mathrm{ml} / \mathrm{L}$.

Foliar spray with moringa extract at the rate of $100 \mathrm{ml} / \mathrm{L}$. Foliar spray with chitosan at the rate of $200 \mathrm{mg} / \mathrm{L}$. 
Active dry yeast were dissolved in water at rate 1 $\mathrm{g} / \mathrm{L}$ followed by adding sugar at ratio $1: 1$ and kept overnight for activation and reproduction of yeast (Spencer et al.,1983).

Moringa leaf extract was prepared according to Culver et al. (2012). The extract was diluted with distilled water at a ratio $1: 32(\mathrm{v} / \mathrm{v})$ and then sprayed directly onto plants.Chitosan powder (Poly-(1.4-B-D-glucopyranosamine) ; 2- Amino- 2-deoxy- (1-4) -B-D-glucopyranan) was prepared by dissolving a proper amount in $5 \%$ acetic acid solution.

Spraying was conducted by hand sprayer (for experimental plots) until saturation point three times after 45, 60 and 75 days from planting date.

Random samples from the experimental soil were obtained at depth of 0 up to $60 \mathrm{~cm}$ pre-planting time to determine the physical and chemical properties as presented in Table (1).

Table 1. The physical and chemical properties of the experimental soil during 2015/2016 and 2016/2017.

\begin{tabular}{|c|c|c|}
\hline \multirow{2}{*}{$\begin{array}{l}\text { Soil properties } \\
\text { Physical analysis. }\end{array}$} & 2015/2016 & 2016/2017 \\
\hline & \multicolumn{2}{|c|}{ Physical analysis: } \\
\hline Coarse sand $(\%)$ & 1.48 & 1.61 \\
\hline Fine sand $\quad(\%)$ & 21.12 & 21.17 \\
\hline$(\%)$ & 25.57 & 25.41 \\
\hline Clay & 49.12 & 49.03 \\
\hline Soil type & Clay & Clay \\
\hline \multicolumn{3}{|l|}{ Chemical analysis: } \\
\hline $\mathrm{CaCO} 3$ & 2.71 & 2.78 \\
\hline Organic matter & 2.80 & 2.78 \\
\hline Total nitrogen & 0.12 & 0.13 \\
\hline Available phosphorus (ppm) & 7.14 & 7.35 \\
\hline Exchangeable potassium (ppm) & 211.00 & 219.00 \\
\hline $\mathrm{pH}$ & 7.91 & 8.01 \\
\hline
\end{tabular}

Garlic cloves were planted on 5th and 8th of October in the first and the second season, respectively. The four sulphur levels occupied the main plots which were subdivided to 4 sub-plots each contained one of the spraying with natural stimulants substances.

Nearly uniform garlic cloves were soaked in running water for $24 \mathrm{~h}$ prior to sowing and hand-planted at 10-12 cm apart on two sides of each row. The sulphur levels were applied as soil application two equal doses, during soil preparation (pre-rowing) and 30 days after planting. All the plants were fertilized with $120 \mathrm{~kg} \mathrm{~N} / \mathrm{fed}$ (ammonium sulphate, 20.5\% N), $90 \mathrm{~kg} \mathrm{P} 2 \mathrm{O} 5 /$ fed (superphosphate, $15.5 \% \mathrm{P} 2 \mathrm{O} 5$ ) and $96 \mathrm{~kg} \mathrm{~K} 2 \mathrm{O} /$ fed (potassium sulphate $48 \% \mathrm{~K} 2 \mathrm{O}$ ) which added in three equal doses 30 , 60 and 90 days after planting. The other cultural practices for garlic commercial production were used according to the instructions laid down by the Ministry of Agriculture, Egypt. The harvesting time was in the first week of April, for both seasons.

\section{Data recorded:}

\section{1- Growth parameters:}

A random sample of five plants was randomly taken from each plot at 120 days after planting to estimate plant height, plant fresh weight $(\mathrm{g})$, plant leaves fresh weight $(\mathrm{g})$, number of leaves/plant, bulbing ratio = $\frac{\text { Plant neck diameter }(\mathrm{cm})}{\text { Bulb diameter }(\mathrm{cm})} \quad$ Mann (1952), plant dry weight (g) and plant leaf area $(\mathrm{cm} 2)$.

\section{2- Yield and its components:}

At harvest time, a random sample (10 bulbs) was randomly taken from each treatment to determine bulb weight $(\mathrm{g})$, bulb diameter $(\mathrm{cm})$, number of cloves/bulb, cloves weight $(\mathrm{g})$. All bulbs of each plot were cured, 15 days after harvest weighted in $\mathrm{kg}$ and converted to record as total yield ( $\mathrm{t} / \mathrm{fed})$.

\section{3- Chemical analysis:}

Samples of the dried cloves were ground, wet digested as described by Hesse (1971) and their nitrogen $(\mathrm{N})$, phosphorus $(\mathrm{P})$, potassium $(\mathrm{K})$ and sulphur (S) contents were determined according to the methods described by Jackson (1967), Black (1965) and Hunter (1984), respectively. Percentage of total soluble solids (TSS\%) and volatile oils (cm3/kg bulbs fresh weight) were determined according to A.O.A.C. (1970)and Guenther (1961), respectively.

\section{4- Storability:}

After curing, random samples $(10 \mathrm{~kg})$ were taken from every experimental unit, stored at room conditions and weight loss percentage was recorded monthly along five months of the storage period. The averages of air temperature and relative humidity in store room were recorded in Table (2).

Table 2. Averages of monthly air temperatures and relative humidity in store room during the storage period in 2016 and 2017 seasons.

\begin{tabular}{lcccc}
\hline \multirow{2}{*}{ Months } & \multicolumn{2}{c}{$\mathbf{2 0 1 6}$} & \multicolumn{2}{c}{$\mathbf{2 0 1 7}$} \\
\cline { 2 - 5 } & Temperature (oC) & Relative humidity (\%) & Temperature (oC) & Relative humidity (\%) \\
\hline April & 15.1 & 56.9 & 16.6 & 58.3 \\
May & 17.6 & 59.3 & 18.1 & 61.6 \\
June & 19.3 & 61.6 & 19.6 & 63.9 \\
July & 21.3 & 66.1 & 22.4 & 65.2 \\
August & 22.9 & 67.8 & 22.9 & 68.1 \\
September & 21.6 & 69.2 & 20.5 & 68.8 \\
October & 18.7 & 70.1 & 18.6 & 70.3 \\
\hline
\end{tabular}

All collected data on plot-basis were subjected to the statistical analysis according to ANOVA for split-plot design as published by Gomez and Gomez (1984), using CoStat Computer Software package. Means of treatments were compared using Duncan's multiple range tests at $5 \%$ level of probability as described by Duncan (1955).

\section{RESULTS AND DISCUSSION}

\section{1-Vegetative growth:}

Effect of sulphur levels:

The data presented in Table (3) show that plant height, number of leaves/plant, plant dry weight and leaf area/plant were significantly increased with increasing 
sulphur levels up to $450 \mathrm{~kg} \mathrm{~S} / \mathrm{fed}$. Also, the bulbing ratio was better with supplying sulphur in both seasons. These results may be due to the beneficial effect of the applied-S as a one of main elements required for plant growth and important in the formation of protein and chlorophylls (Marschner, 2012). These results are in agreement with those of El-Morsy (2005), Hore et al. (2014), Assefa et al. (2015), Youssifet al. (2015), Babaleshwar (2017), Patidar et al. (2017) and Shete et al. (2018), they found that plant growth increased significantly with increasing the rate of applied sulphur.

Table 3. Vegetative growth characters of garlic plants as affected by sulphur levels, natural stimulant substances and their interactions at 120 day after planting during 2015/2016 (S1) and 2016/2017 (S2) seasons.

\begin{tabular}{|c|c|c|c|c|c|c|c|c|c|c|c|}
\hline \multirow{2}{*}{\multicolumn{2}{|c|}{$\begin{array}{l}\text { Characters } \\
\text { Treatments }\end{array}$}} & \multicolumn{2}{|c|}{$\begin{array}{l}\text { Plant height } \\
\text { (cm) }\end{array}$} & \multicolumn{2}{|c|}{$\begin{array}{l}\text { Number of } \\
\text { leaves/plant }\end{array}$} & \multicolumn{2}{|c|}{$\begin{array}{c}\text { Bulbing } \\
\text { ratio }\end{array}$} & \multicolumn{2}{|c|}{$\begin{array}{l}\text { Plant dry weight } \\
\text { (g) }\end{array}$} & \multicolumn{2}{|c|}{$\begin{array}{l}\text { Leaf area/plant } \\
(\mathrm{cm} 2)\end{array}$} \\
\hline & & S1 & S2 & S1 & S2 & S1 & S2 & S1 & S2 & S1 & S2 \\
\hline \multicolumn{12}{|c|}{ Sulphur levels: } \\
\hline \multicolumn{2}{|l|}{$150 \mathrm{~kg} \mathrm{~S} / \mathrm{fed}$} & $79.60 \mathrm{~b}$ & $80.45 \mathrm{ab}$ & $10.57 \mathrm{a}$ & $10.43 \mathrm{a}$ & $0.38 \mathrm{a}$ & $0.38 \mathrm{a}$ & $41.09 \mathrm{~b}$ & $41.51 \mathrm{bc}$ & $2042.0 \mathrm{bc}$ & $2005.0 \mathrm{c}$ \\
\hline \multicolumn{2}{|l|}{$300 \mathrm{~kg} \mathrm{~S} / \mathrm{fed}$} & $80.45 \mathrm{~b}$ & $80.67 \mathrm{ab}$ & $10.69 \mathrm{a}$ & $10.79 \mathrm{a}$ & $0.36 \mathrm{~b}$ & $0.36 \mathrm{~b}$ & $42.76 \mathrm{~b}$ & $43.66 \mathrm{~b}$ & $2237.0 \mathrm{ab}$ & $2409.0 \mathrm{~b}$ \\
\hline \multicolumn{2}{|l|}{$450 \mathrm{~kg} \mathrm{~S} / \mathrm{fed}$} & $82.14 \mathrm{a}$ & $83.34 \mathrm{a}$ & $11.03 \mathrm{a}$ & $11.13 \mathrm{a}$ & $0.32 \mathrm{c}$ & $0.31 \mathrm{c}$ & $47.07 \mathrm{a}$ & $46.74 \mathrm{a}$ & $2416.0 \mathrm{a}$ & $2569.0 \mathrm{a}$ \\
\hline \multicolumn{12}{|c|}{ Natural stimulant substances } \\
\hline \multicolumn{2}{|c|}{ Control } & $77.37 \mathrm{~d}$ & $78.50 \mathrm{~d}$ & $9.98 \mathrm{~d}$ & $9.56 \mathrm{~d}$ & $0.38 \mathrm{a}$ & $0.38 \mathrm{a}$ & $40.23 \mathrm{~d}$ & $41.29 \mathrm{~d}$ & $1985.0 \mathrm{c}$ & $2075.0 \mathrm{~d}$ \\
\hline \multicolumn{2}{|c|}{ Yeast (100 ml/L) } & $79.41 \mathrm{c}$ & $80.37 \mathrm{c}$ & $10.41 \mathrm{c}$ & $10.76 \mathrm{c}$ & $0.36 \mathrm{~b}$ & $0.36 \mathrm{~b}$ & $41.50 \mathrm{c}$ & $42.63 \mathrm{c}$ & $2050.0 \mathrm{c}$ & $2162.0 \mathrm{c}$ \\
\hline \multicolumn{2}{|c|}{ Moringa $(100$ ml/L) } & $81.18 \mathrm{~b}$ & $81.57 \mathrm{~b}$ & $10.69 \mathrm{~b}$ & $10.98 \mathrm{~b}$ & $0.35 \mathrm{c}$ & $0.35 \mathrm{c}$ & $42.95 \mathrm{~b}$ & $43.61 \mathrm{~b}$ & $2194.0 \mathrm{~b}$ & $2214.0 \mathrm{~b}$ \\
\hline \multicolumn{2}{|c|}{ Chitosan $(200 \mathrm{mg} / \mathrm{L})$} & $83.27 \mathrm{a}$ & $83.25 \mathrm{a}$ & $11.05 \mathrm{a}$ & $11.03 \mathrm{a}$ & $0.35 \mathrm{~d}$ & $0.34 \mathrm{~d}$ & $44.57 \mathrm{a}$ & $44.43 \mathrm{a}$ & $2326.0 \mathrm{a}$ & $2286.0 \mathrm{a}$ \\
\hline \multicolumn{12}{|c|}{ Interactions: } \\
\hline \multicolumn{12}{|l|}{ S-levels X } \\
\hline \multirow{4}{*}{ Control } & Control & $74.80 \mathrm{i}$ & $76.70 \mathrm{j}$ & $9.20 \mathrm{i}$ & $8.96 \mathrm{~h}$ & $0.41 \mathrm{a}$ & $0.41 \mathrm{a}$ & $36.41 \mathrm{i}$ & $38.21 \mathrm{j}$ & $1727.0 \mathrm{~h}$ & $1688.0 \mathrm{k}$ \\
\hline & Yeast & $78.43 \mathrm{f}-\mathrm{h}$ & $77.90 \mathrm{ij}$ & $9.76 \mathrm{~h}$ & $9.26 \mathrm{~h}$ & $0.39 \mathrm{~b}$ & $0.39 \mathrm{~b}$ & $37.51 \mathrm{i}$ & $40.03 \mathrm{i}$ & $1757.0 \mathrm{~h}$ & $1746.0 \mathrm{jk}$ \\
\hline & Moringa & $80.36 \mathrm{~d}-\mathrm{g}$ & $79.93 \mathrm{~h}$ & $10.10 \mathrm{gh}$ & $9.83 \mathrm{~g}$ & $0.38 \mathrm{~b}-\mathrm{d}$ & $0.38 \mathrm{~b}-\mathrm{d}$ & $38.89 \mathrm{~h}$ & $40.73 \mathrm{hi}$ & $1914.0 \mathrm{fg}$ & $1834.0 \mathrm{ij}$ \\
\hline & Chitosan & $82.59 \mathrm{a}-\mathrm{d}$ & $82.36 \mathrm{~b}-\mathrm{d}$ & $10.33 \mathrm{e}-\mathrm{g}$ & $10.20 \mathrm{fg}$ & $0.37 \mathrm{de}$ & $0.37 \mathrm{c}-\mathrm{e}$ & $40.49 \mathrm{fg}$ & $41.19 \mathrm{~g}-\mathrm{i}$ & $2034.0 \mathrm{ef}$ & $1851.0 \mathrm{i}$ \\
\hline \multirow{4}{*}{$\begin{array}{l}150 \mathrm{~kg} \\
\mathrm{~S} / \mathrm{fed}\end{array}$} & Control & $76.70 \mathrm{hi}$ & $78.06 \mathrm{i}$ & $10.10 \mathrm{gh}$ & $10.16 \mathrm{fg}$ & $0.39 \mathrm{~b}$ & $0.39 \mathrm{~b}$ & $39.26 \mathrm{gh}$ & $39.95 \mathrm{i}$ & $1835.0 \mathrm{gh}$ & $1900.0 \mathrm{hi}$ \\
\hline & Yeast & $78.43 \mathrm{f}-\mathrm{h}$ & $80.63 \mathrm{f}-\mathrm{h}$ & $10.53 \mathrm{c}-\mathrm{g}$ & $10.70 \mathrm{de}$ & $0.38 \mathrm{bc}$ & $0.38 \mathrm{bc}$ & $40.33 \mathrm{fg}$ & $41.36 \mathrm{~g}-\mathrm{i}$ & $1938.0 \mathrm{fg}$ & $1962.0 \mathrm{gh}$ \\
\hline & Moringa & $80.43 \mathrm{c}-\mathrm{f}$ & $81.43 \mathrm{~d}-\mathrm{f}$ & $10.66 \mathrm{~b}-\mathrm{f}$ & $10.86 \mathrm{~cd}$ & $0.38 \mathrm{~b}-\mathrm{d}$ & $0.38 \mathrm{~b}-\mathrm{d}$ & $41.54 \mathrm{ef}$ & $42.08 \mathrm{f}-\mathrm{h}$ & $2116.0 \mathrm{e}$ & $2036.0 \mathrm{fg}$ \\
\hline & Chitosan & $82.80 \mathrm{a}-\mathrm{c}$ & $82.56 \mathrm{~b}-\mathrm{d}$ & $11.00 \mathrm{bc}$ & $11.33 \mathrm{ab}$ & $0.37 \mathrm{de}$ & $0.37 \mathrm{ef}$ & $43.23 \mathrm{~d}$ & $42.67 \mathrm{fg}$ & $2281.0 \mathrm{~cd}$ & $2122.0 \mathrm{f}$ \\
\hline \multirow{4}{*}{$\begin{array}{l}300 \mathrm{~kg} \\
\mathrm{~S} / \text { fed }\end{array}$} & Control & $78.00 \mathrm{gh}$ & $78.16 \mathrm{i}$ & $10.20 \mathrm{f}-\mathrm{h}$ & $10.36 \mathrm{ef}$ & $0.38 \mathrm{bc}$ & $0.38 \mathrm{bcd}$ & $40.56 \mathrm{f}$ & $42.44 \mathrm{fg}$ & $2062.0 \mathrm{ef}$ & $2231.0 \mathrm{e}$ \\
\hline & Yeast & $79.80 \mathrm{e}-\mathrm{g}$ & $80.10 \mathrm{gh}$ & $10.56 \mathrm{c}-\mathrm{g}$ & $10.86 \mathrm{~cd}$ & $0.37 \mathrm{~cd}$ & $0.37 \mathrm{de}$ & $42.28 \mathrm{de}$ & 43.32ef & $2133.0 \mathrm{de}$ & $2407.0 \mathrm{~d}$ \\
\hline & Moringa & $80.73 \mathrm{c}-\mathrm{f}$ & $81.33 \mathrm{~d}-\mathrm{g}$ & $10.90 \mathrm{~b}-\mathrm{d}$ & $11.16 \mathrm{a}-\mathrm{c}$ & 0.36 ef & $0.36 \mathrm{f}$ & $43.26 \mathrm{~d}$ & $44.32 \mathrm{de}$ & $2316.0 \mathrm{bc}$ & $2483.0 \mathrm{~cd}$ \\
\hline & Chitosan & $83.26 \mathrm{ab}$ & $82.23 \mathrm{c}-\mathrm{e}$ & $11.10 \mathrm{~b}$ & $11.53 \mathrm{a}$ & $0.35 \mathrm{f}$ & $0.34 \mathrm{~g}$ & $44.96 \mathrm{c}$ & $44.79 \mathrm{~cd}$ & $2439.0 \mathrm{ab}$ & $2516.0 \mathrm{c}$ \\
\hline \multirow{4}{*}{$\begin{array}{l}450 \mathrm{~kg} \\
\mathrm{~S} / \text { fed }\end{array}$} & Control & $79.93 \mathrm{e}-\mathrm{g}$ & $81.0 \mathrm{e}-\mathrm{h}$ & $10.43 \mathrm{~d}-\mathrm{g}$ & $10.46 \mathrm{~d}-\mathrm{f}$ & $0.33 \mathrm{~g}$ & $0.33 \mathrm{~h}$ & $44.70 \mathrm{c}$ & $44.57 \mathrm{c}-\mathrm{e}$ & $2315.0 \mathrm{bc}$ & $2480.0 \mathrm{~cd}$ \\
\hline & Yeast & 81.00 b-e & $82.86 \mathrm{bc}$ & $10.80 \mathrm{~b}-\mathrm{e}$ & $10.90 \mathrm{~b}-\mathrm{d}$ & $0.32 \mathrm{gh}$ & $0.31 \mathrm{i}$ & $45.87 \mathrm{c}$ & $46.04 \mathrm{bc}$ & $2370.0 \mathrm{bc}$ & $2532.0 \mathrm{bc}$ \\
\hline & Moringa & $83.20 \mathrm{ab}$ & $83.60 \mathrm{~b}$ & $11.10 \mathrm{~b}$ & $11.30 \mathrm{a}-\mathrm{c}$ & $0.31 \mathrm{hi}$ & $0.30 \mathrm{ij}$ & $48.11 \mathrm{~b}$ & $47.29 \mathrm{~b}$ & $2430.0 \mathrm{a}-\mathrm{c}$ & $2610.0 \mathrm{ab}$ \\
\hline & Chitosan & $84.43 \mathrm{a}$ & $85.83 \mathrm{a}$ & $11.80 \mathrm{a}$ & $11.46 \mathrm{a}$ & $0.31 \mathrm{i}$ & $0.30 \mathrm{j}$ & $49.60 \mathrm{a}$ & $49.06 \mathrm{a}$ & $2551.0 \mathrm{a}$ & $2654.0 \mathrm{a}$ \\
\hline
\end{tabular}

Means followed by the same letter in column are not significantly differed according to Duncan's Multiple Range Test at 5 \% level of probability. NSS=Natural stimulant substances

Effect of foliar spray with some natural stimulant substances:

Data in Table (3) also, reveal that foliar spray with some natural stimulant substances i.e. untreated (control treatment), yeast extract, moringa extract and chitosan was associated significant effect on vegetative growth characteristics (plant height, number of leaves/plant, bulbing ratio, plant dry weight and leaf area/plant in both seasons. Plants sprayed with chitosan exceeded other foliar spray with some natural stimulant substances and produced the highest values of all studied vegetative growth characteristics, this treatment enhanced bulbing ratioin both growing seasons also. These results may be due to the role of chitosan, which has become a useful appreciated compound due to its fungicidal effects and elicitation of defense mechanisms in plant tissues. In addition, the role of fresh moringa leaves extract, which have been shown to have high zeatin content, where zeatin is one form of the most common forms of naturally occurring cytokinin in plants. Also, yeast extract had stimulatory effects on cell division and enlargement, protein and nucleic acid synthesis and chlorophyll formation. These results were parallel with those reported by El-Morsy et al. (2011),
Fawzy et al. (2012), Shalaby and El-Ramady (2014), Ahmed (2015) and Hegazi et al. (2016).

Effect of interaction between S-levels and foliar spray with some natural stimulant substances:

It is obvious from the same data in Table (3) that all parameters of vegetative growth characteristics were affected by interaction between sulphur fertilizer levels and foliar spray with some natural stimulant substances. In general, plants received $\mathrm{S}$ at $450 \mathrm{~kg} / \mathrm{fed}$ and sprayed chitosan gave the highest values of plant growth parameters and enhanced bulbing ratio in both seasons, followed by the same level of sulpher and foliar spray with moringa extract.

\section{2- Yield and its components:}

Effect of sulphur levels:

Data illustrated in Table (4) show the effect of sulphur levels on yield and its components of garlic. Such data indicates that the sulphur application improved yield and its components comparing with the control treatment. Total yield/fed, bulb weight, bulb diameter and cloves weight were significantly increased with increasing the sulphur application level up to $450 \mathrm{~kg} \mathrm{~S} / \mathrm{fed}$ in both seasons. Concerning number of cloves/bulb, it was 
significantly decreased with increasing sulphur fertilizer levels up to $450 \mathrm{~kg} \mathrm{~S} / \mathrm{fed}$ in both seasons. The positive effect of this sulphur level may be due to lowering soil $\mathrm{pH}$ factor that improved soil chemical properties and increased the availability of certain plant nutrients. The obtained results are in accordance with those of El-Morsy (2005), Hore et al. (2014), Assefa et al. (2015), Youssif et al. (2015), Babaleshwar et al. (2017), and Shete et al. (2018) stated that total yield and its components were increased significantly with increasing sulphur level.

Table 4. Total yield and its components as affected by sulphur levels, natural stimulant substances and their interactions at 120 day after planting during 2015/2016 (S1) and 2016/2017 (S2) seasons.

\begin{tabular}{|c|c|c|c|c|c|c|c|c|c|c|}
\hline \multirow{2}{*}{$\begin{array}{l}\text { Characters } \\
\text { Treatments }\end{array}$} & \multicolumn{2}{|c|}{$\begin{array}{c}\text { Total yield } \\
\text { (t/fed) }\end{array}$} & \multicolumn{2}{|c|}{$\begin{array}{l}\text { Bulb weight } \\
\text { (g) }\end{array}$} & \multicolumn{2}{|c|}{$\begin{array}{l}\text { Bulb diameter } \\
(\mathrm{cm})\end{array}$} & \multicolumn{2}{|c|}{$\begin{array}{l}\text { Number of } \\
\text { cloves/bulb }\end{array}$} & \multicolumn{2}{|c|}{$\begin{array}{c}\text { Cloves weight } \\
\text { (g) }\end{array}$} \\
\hline & S1 & S2 & S1 & S2 & S1 & S2 & S1 & S2 & S1 & $\mathbf{S 2}$ \\
\hline \multicolumn{11}{|l|}{ Sulphur levels: } \\
\hline Control & $5.44 \mathrm{~d}$ & $5.44 \mathrm{~d}$ & $49.45 \mathrm{~d}$ & $48.50 \mathrm{c}$ & $4.80 \mathrm{~d}$ & $4.07 \mathrm{c}$ & $16.66 \mathrm{a}$ & $14.76 \mathrm{a}$ & $3.02 \mathrm{c}$ & $2.90 \mathrm{~d}$ \\
\hline $150 \mathrm{~kg} \mathrm{~S} / \mathrm{fed}$ & $6.64 \mathrm{c}$ & $6.50 \mathrm{c}$ & $54.47 \mathrm{c}$ & $56.10 \mathrm{~b}$ & $5.21 \mathrm{c}$ & $4.50 \mathrm{~b}$ & $15.50 \mathrm{~b}$ & $14.74 \mathrm{a}$ & $3.22 \mathrm{bc}$ & $3.35 \mathrm{c}$ \\
\hline $300 \mathrm{~kg} \mathrm{~S} / \mathrm{fed}$ & $6.81 \mathrm{~b}$ & $6.56 \mathrm{~b}$ & $58.96 \mathrm{~b}$ & $59.25 \mathrm{~b}$ & $5.39 \mathrm{~b}$ & $4.82 \mathrm{~b}$ & $15.41 \mathrm{~b}$ & $14.25 \mathrm{~b}$ & $3.37 \mathrm{~b}$ & $3.63 \mathrm{~b}$ \\
\hline $450 \mathrm{~kg} \mathrm{~S} / \mathrm{fed}$ & $7.72 \mathrm{a}$ & $7.32 \mathrm{a}$ & $61.55 \mathrm{a}$ & $67.24 \mathrm{a}$ & $5.66 \mathrm{a}$ & $5.51 \mathrm{a}$ & $15.00 \mathrm{~b}$ & $12.83 \mathrm{c}$ & $3.92 \mathrm{a}$ & $4.11 \mathrm{a}$ \\
\hline \multicolumn{11}{|c|}{ Natural stimulant substances: } \\
\hline Control & $6.36 \mathrm{~d}$ & $6.18 \mathrm{~d}$ & $51.45 \mathrm{~d}$ & $54.80 \mathrm{c}$ & $4.89 \mathrm{~d}$ & $4.45 \mathrm{~d}$ & $16.50 \mathrm{a}$ & $14.91 \mathrm{a}$ & $2.96 \mathrm{~b}$ & $3.05 \mathrm{~d}$ \\
\hline Yeast $(100 \mathrm{ml} / \mathrm{L})$ & $6.65 \mathrm{c}$ & $6.33 \mathrm{c}$ & $54.58 \mathrm{c}$ & $57.22 \mathrm{~b}$ & $5.18 \mathrm{c}$ & $4.70 \mathrm{c}$ & $16.25 \mathrm{a}$ & $14.33 \mathrm{~b}$ & $3.10 \mathrm{~b}$ & $3.27 \mathrm{c}$ \\
\hline Moringa $(100 \mathrm{ml} / \mathrm{L})$ & $6.76 \mathrm{~b}$ & $6.58 \mathrm{~b}$ & $57.40 \mathrm{~b}$ & $58.22 \mathrm{~b}$ & $5.40 \mathrm{~b}$ & $4.82 \mathrm{~b}$ & $15.25 \mathrm{~b}$ & $13.91 \mathrm{~b}$ & $3.57 \mathrm{a}$ & $3.68 \mathrm{~b}$ \\
\hline Chitosan (200 mg/L) & $6.85 \mathrm{a}$ & $6.73 \mathrm{a}$ & $61.00 \mathrm{a}$ & $60.85 \mathrm{a}$ & $5.60 \mathrm{a}$ & $4.95 \mathrm{a}$ & $14.58 \mathrm{c}$ & $13.41 \mathrm{c}$ & $3.90 \mathrm{a}$ & $4.00 \mathrm{a}$ \\
\hline
\end{tabular}

\begin{tabular}{|c|c|c|c|c|c|c|c|c|c|c|c|}
\hline \multicolumn{12}{|l|}{ Interactions: } \\
\hline S-levels X & NSS & & & & & & & & & & \\
\hline \multirow{4}{*}{ Control } & Control & 5.321 & $5.29 \mathrm{o}$ & $45.33 \mathrm{i}$ & $44.33 \mathrm{~g}$ & $4.16 \mathrm{k}$ & $3.83 \mathrm{k}$ & $16.00 \mathrm{bc}$ & $15.65 \mathrm{a}$ & $2.83 \mathrm{de}$ & $2.30 \mathrm{i}$ \\
\hline & Yeast & 5.341 & $5.38 \mathrm{n}$ & $47.60 \mathrm{~h}$ & $47.86 \mathrm{f}$ & $4.80 \mathrm{j}$ & $4.03 \mathrm{j}$ & $16.00 \mathrm{bc}$ & $14.66 \mathrm{bc}$ & $2.86 \mathrm{de}$ & $2.50 \mathrm{i}$ \\
\hline & Moringa & $5.49 \mathrm{k}$ & $5.46 \mathrm{~m}$ & $50.93 \mathrm{fg}$ & $49.06 \mathrm{f}$ & $5.03 \mathrm{~h}-\mathrm{j}$ & $4.20 \mathrm{ij}$ & $15.00 \mathrm{c}-\mathrm{e}$ & $14.66 \mathrm{bc}$ & $2.03 \mathrm{~cd}$ & $3.26 \mathrm{f}-\mathrm{h}$ \\
\hline & Chitosan & $5.62 \mathrm{j}$ & 5.631 & $53.93 \mathrm{e}$ & $52.73 \mathrm{e}$ & $5.23 \mathrm{e}-\mathrm{h}$ & $4.23 \mathrm{i}$ & $15.00 \mathrm{c}-\mathrm{e}$ & $14.00 \mathrm{~cd}$ & $3.36 \mathrm{~b}-\mathrm{d}$ & $3.56 \mathrm{~d}-\mathrm{e}$ \\
\hline \multirow{4}{*}{$\begin{array}{l}150 \mathrm{~kg} \\
\mathrm{~S} / \text { fed }\end{array}$} & Control & $5.94 \mathrm{i}$ & $5.98 \mathrm{k}$ & $49.50 \mathrm{gh}$ & $52.56 \mathrm{e}$ & $4.86 \mathrm{ij}$ & $4.20 \mathrm{ij}$ & $18.01 \mathrm{a}$ & $15.67 \mathrm{a}$ & $2.96 \mathrm{de}$ & $3.13 \mathrm{~h}$ \\
\hline & Yeast & $6.81 \mathrm{~g}$ & $6.34 \mathrm{j}$ & 52.03 ef & $55.50 \mathrm{~d}$ & $5.10 \mathrm{~g}-\mathrm{i}$ & $4.43 \mathrm{~h}$ & $17.99 \mathrm{a}$ & $15.00 \mathrm{ab}$ & $2.03 \mathrm{~cd}$ & $3.33 \mathrm{e}-\mathrm{h}$ \\
\hline & Moringa & $6.89 \mathrm{f}$ & $6.80 \mathrm{f}$ & $56.46 \mathrm{~d}$ & $56.06 \mathrm{~d}$ & $5.36 \mathrm{~d}-\mathrm{f}$ & $4.63 \mathrm{fg}$ & $15.33 \mathrm{c}-\mathrm{e}$ & $14.33 \mathrm{~b}-\mathrm{d}$ & $3.36 \mathrm{~b}-\mathrm{d}$ & $3.40 \mathrm{~d}-\mathrm{h}$ \\
\hline & Chitosan & $6.94 \mathrm{e}$ & $6.90 \mathrm{e}$ & $59.90 \mathrm{c}$ & $60.30 \mathrm{bc}$ & $5.53 \mathrm{~cd}$ & $4.67 \mathrm{ef}$ & $15.33 \mathrm{c}-\mathrm{e}$ & $14.00 \mathrm{~cd}$ & $3.53 \mathrm{~b}-\mathrm{d}$ & $3.53 \mathrm{c}-\mathrm{f}$ \\
\hline \multirow{4}{*}{$\begin{array}{l}300 \mathrm{~kg} \\
\mathrm{~S} / \mathrm{fed}\end{array}$} & Control & $6.65 \mathrm{~h}$ & $6.47 \mathrm{i}$ & $53.96 \mathrm{e}$ & $56.10 \mathrm{~d}$ & $5.16 \mathrm{f}-\mathrm{h}$ & $4.46 \mathrm{gh}$ & $17.00 \mathrm{ab}$ & $14.66 \mathrm{bc}$ & $3.10 \mathrm{~cd}$ & $3.20 \mathrm{gh}$ \\
\hline & Yeast & $6.79 \mathrm{~g}$ & $6.48 \mathrm{i}$ & $58.50 \mathrm{~cd}$ & $58.60 \mathrm{c}$ & $5.33 \mathrm{~d}-\mathrm{g}$ & $4.90 \mathrm{de}$ & $15.33 \mathrm{c}-\mathrm{e}$ & $14.66 \mathrm{bc}$ & $2.24 \mathrm{e}$ & $3.46 \mathrm{~d}-\mathrm{g}$ \\
\hline & Moringa & $6.89 \mathrm{f}$ & $6.54 \mathrm{~h}$ & $59.26 \mathrm{c}$ & $60.30 \mathrm{bc}$ & $5.43 \mathrm{c}-\mathrm{e}$ & $4.93 \mathrm{de}$ & $15.00 \mathrm{c}-\mathrm{e}$ & $14.00 \mathrm{~cd}$ & $3.86 \mathrm{ab}$ & $3.63 \mathrm{~cd}$ \\
\hline & Chitosan & $6.92 \mathrm{e}$ & $6.76 \mathrm{~g}$ & $64.13 \mathrm{ab}$ & $62.03 \mathrm{~b}$ & $5.63 \mathrm{bc}$ & $5.00 \mathrm{~d}$ & 14.33 ef & $13.66 \mathrm{de}$ & $4.30 \mathrm{a}$ & $4.23 \mathrm{~b}$ \\
\hline \multirow{4}{*}{$\begin{array}{l}450 \mathrm{~kg} \\
\mathrm{~S} / \mathrm{fed}\end{array}$} & Control & $7.54 \mathrm{~d}$ & $6.98 \mathrm{~d}$ & $57.00 \mathrm{~d}$ & $66.23 \mathrm{a}$ & $5.36 \mathrm{~d}-\mathrm{f}$ & $5.30 \mathrm{c}$ & $16.00 \mathrm{bc}$ & $13.66 \mathrm{de}$ & $3.53 \mathrm{~b}-\mathrm{d}$ & $3.56 \mathrm{c}-\mathrm{e}$ \\
\hline & Yeast & $7.68 \mathrm{c}$ & $7.15 \mathrm{c}$ & $60.20 \mathrm{c}$ & $66.93 \mathrm{a}$ & $5.50 \mathrm{~cd}$ & $5.43 b c$ & $15.66 \mathrm{~cd}$ & 13.00 ef & $3.73 \mathrm{a}-\mathrm{c}$ & $3.80 \mathrm{c}$ \\
\hline & Moringa & $7.77 \mathrm{~b}$ & $7.51 \mathrm{~b}$ & $62.93 \mathrm{~b}$ & $67.46 \mathrm{a}$ & $5.80 \mathrm{ab}$ & $5.53 \mathrm{~b}$ & $14.66 \mathrm{~d}-\mathrm{f}$ & $12.66 \mathrm{fg}$ & $4.03 \mathrm{ab}$ & $4.43 \mathrm{ab}$ \\
\hline & Chitosan & $7.91 \mathrm{a}$ & $7.64 \mathrm{a}$ & $66.06 \mathrm{a}$ & $68.33 \mathrm{a}$ & $6.00 \mathrm{a}$ & $5.80 \mathrm{a}$ & $13.66 \mathrm{f}$ & $12.00 \mathrm{~g}$ & $4.40 \mathrm{a}$ & $4.66 \mathrm{a}$ \\
\hline
\end{tabular}

Means followed by the same letter in column are not significantly differed according to Duncan's Multiple Range Test at $5 \%$ level of probability. NSS $=$ Natural stimulant substances

\section{Effect of foliar spray with some natural stimulant} substances:

Data in Table (4) indicate that spray with natural stimulant substances was more effective in total yield and its components than the control treatment. Plants sprayed with chitosan gave the highest yield, bulb weight, bulb diameter and clove weight, whereas number of cloves per bulb was reduced. These increases might be ascribed to the favourable role of natural stimulants like chitosan, moringa extract and yeast extract as mentioned formerly in improving plant growth characteristics, which reflected on increasing yield and its components. Similar results were reported by El-Morsy et al. (2011), Shalaby and ElRamady (2014), Ahmed (2015) and Hegazi et al. (2016).

Effect of interaction between S-levels and foliar spray with some natural stimulant substances:

It is obvious from data in Table (4) that there were significant interactions between sulphur fertilizer levels and foliar spray with some natural stimulant substances on yield and its components (bulb weight, bulb diameter, number of cloves and cloves weight) of garlic in both seasons. In general, plants fertilized by $450 \mathrm{~kg}$ S/fed and sprayed with chitosan produced the highest values of yield and its components. Moreover, number of cloves per bulb trait was decreased. These results coincide with those of Ahmed (2015).

\section{3- Chemical constituents:}

\section{Effect of sulphur levels:}

Data in Table (5) clear show that the sulphur application levels had a significant effect on nitrogen, phosphorus, potassium, sulphur, volatile oils and total soluble solids (TSS \%) contents in garlic cloves at harvest in both seasons. All elements concentrations in cloves were significantly increased with increasing sulphur application level in both seasons. Application of $450 \mathrm{~kg} \mathrm{~S} /$ fed gave the highest records, followed by the $300 \mathrm{~kg} \mathrm{~S} /$ fed in both seasons. These results may be related to the positive effect of sulphur on the availability of some nutrient elements, which was reflected on plant uptake and plant growth (Dahdouh et al., 1993; Mehana, 1994 and Mehana and Farag, 2000). These results are in agreement with those of El-Morsy (2005) and Shete et al. (2018). 
Table 5. Chemical constituents in garlic bulbs as affected by sulphur levels, natural stimulant substances and their interactions at 120 day after planting during 2015/2016 (S1) and 2016/2017 (S2) seasons.

\begin{tabular}{|c|c|c|c|c|c|c|c|c|c|c|c|c|c|}
\hline \multirow{2}{*}{\multicolumn{2}{|c|}{$\begin{array}{l}\text { Characters } \\
\text { Treatments }\end{array}$}} & \multicolumn{2}{|c|}{$\begin{array}{l}\mathrm{N} \\
(\%)\end{array}$} & \multicolumn{2}{|c|}{$\begin{array}{l}P \\
(\%)\end{array}$} & \multicolumn{2}{|c|}{$\begin{array}{l}\mathrm{K} \\
(\%)\end{array}$} & \multicolumn{2}{|c|}{$\begin{array}{l} \\
(\%)\end{array}$} & \multicolumn{2}{|c|}{$\begin{array}{c}\text { Volatile oils } \\
\text { (cm3/kg F.W) }\end{array}$} & \multicolumn{2}{|c|}{$\begin{array}{l}\text { TSS } \\
(\%)\end{array}$} \\
\hline & & S1 & S2 & S1 & S2 & S1 & S2 & S1 & $\mathbf{S 2}$ & S1 & $\mathbf{S 2}$ & S1 & S2 \\
\hline \multicolumn{14}{|c|}{ Sulphur levels: } \\
\hline \multicolumn{2}{|l|}{ Control } & $1.36 \mathrm{~d}$ & $1.47 \mathrm{~d}$ & $0.42 \mathrm{c}$ & $0.46 \mathrm{c}$ & $2.34 \mathrm{c}$ & $2.43 \mathrm{~d}$ & $0.75 \mathrm{~d}$ & $0.73 \mathrm{~d}$ & $0.34 \mathrm{c}$ & $0.36 \mathrm{~d}$ & $31.54 \mathrm{~d}$ & $33.95 \mathrm{c}$ \\
\hline \multicolumn{2}{|c|}{$150 \mathrm{~kg} \mathrm{~S} / \mathrm{fed}$} & $1.43 \mathrm{c}$ & $1.53 \mathrm{c}$ & $0.44 \mathrm{c}$ & $0.48 \mathrm{c}$ & $2.35 \mathrm{c}$ & $2.47 \mathrm{c}$ & $0.78 \mathrm{c}$ & $0.80 \mathrm{c}$ & $0.41 \mathrm{~b}$ & $0.42 \mathrm{c}$ & $33.20 \mathrm{c}$ & $.66 \mathrm{c}$ \\
\hline \multicolumn{2}{|c|}{$300 \mathrm{~kg} \mathrm{~S} / \mathrm{fed}$} & $1.49 \mathrm{~b}$ & $1.55 \mathrm{~b}$ & $0.46 \mathrm{~b}$ & $0.53 \mathrm{~b}$ & $2.39 \mathrm{~b}$ & $2.53 \mathrm{~b}$ & $0.79 \mathrm{~b}$ & $0.89 \mathrm{~b}$ & $0.44 \mathrm{a}$ & $0.45 \mathrm{~b}$ & $35.04 \mathrm{~b}$ & $36.62 \mathrm{~d}$ \\
\hline \multicolumn{2}{|c|}{$450 \mathrm{~kg} \mathrm{~S} / \mathrm{fed}$} & $1.53 \mathrm{a}$ & $1.59 \mathrm{a}$ & $0.53 \mathrm{a}$ & $0.58 \mathrm{a}$ & $2.47 \mathrm{a}$ & $2.59 \mathrm{a}$ & $0.80 \mathrm{a}$ & $1.00 \mathrm{a}$ & $0.45 \mathrm{a}$ & $0.46 \mathrm{a}$ & $37.33 \mathrm{a}$ & $39.83 \mathrm{a}$ \\
\hline \multicolumn{14}{|c|}{ Natural stimulant substances: } \\
\hline \multicolumn{2}{|l|}{ Control } & $1.43 \mathrm{~d}$ & $1.51 \mathrm{~d}$ & $0.43 \mathrm{~d}$ & $0.49 \mathrm{~d}$ & $2.36 \mathrm{~d}$ & $2.48 \mathrm{~d}$ & $0.65 \mathrm{~d}$ & $0.82 \mathrm{~d}$ & $0.38 \mathrm{~d}$ & $0.38 \mathrm{~d}$ & $33.41 \mathrm{~d}$ & $34.50 \mathrm{~d}$ \\
\hline \multicolumn{2}{|c|}{ Yeast $(100 \mathrm{ml} / \mathrm{L})$} & $5 \mathrm{c}$ & $1.53 \mathrm{c}$ & $0.45 \mathrm{c}$ & $0.51 \mathrm{c}$ & $2.38 \mathrm{c}$ & $2.50 \mathrm{c}$ & $0.72 \mathrm{c}$ & $0.84 \mathrm{c}$ & $0.37 \mathrm{c}$ & $0.41 \mathrm{c}$ & $34.00 \mathrm{c}$ & $35.66 \mathrm{c}$ \\
\hline \multicolumn{2}{|c|}{ Moringa $(100 \mathrm{ml} / \mathrm{L})$} & $1.46 \mathrm{~b}$ & $1.54 \mathrm{~b}$ & $0.47 \mathrm{~b}$ & $0.52 \mathrm{~b}$ & $2.40 \mathrm{~b}$ & $2.51 \mathrm{~b}$ & $0.82 \mathrm{~b}$ & $0.86 b$ & $0.43 \mathrm{~b}$ & $0.44 \mathrm{~b}$ & $34.50 \mathrm{~b}$ & $37.00 \mathrm{~b}$ \\
\hline \multicolumn{2}{|c|}{ Chitosan $(200 \mathrm{mg} / \mathrm{L})$} & $1.48 \mathrm{a}$ & $1.55 \mathrm{a}$ & $0.49 \mathrm{a}$ & $0.53 \mathrm{a}$ & $2.42 \mathrm{a}$ & $2.52 \mathrm{a}$ & $0.93 \mathrm{a}$ & $0.90 \mathrm{a}$ & $0.46 \mathrm{a}$ & $0.46 \mathrm{a}$ & $35.20 \mathrm{a}$ & $37.91 \mathrm{a}$ \\
\hline \multicolumn{14}{|c|}{ Interaction } \\
\hline \multirow[t]{2}{*}{ S-levels } & $\mathrm{X} \quad \mathrm{NSS}$ & & & & & & & & & & & & \\
\hline & Control & $1.34 \mathrm{~m}$ & $1.45 \mathrm{k}$ & $0.39 \mathrm{j}$ & $0.45 n$ & $2.32 \mathrm{k}$ & 2.421 & $0.63 \mathrm{p}$ & 0.711 & 0.361 & $0.37 \mathrm{i}$ & 31.001 & $32.66 \mathrm{~g}$ \\
\hline \multirow{3}{*}{ Control } & Yeast & 1.361 & $1.46 \mathrm{k}$ & $0.41 \mathrm{i}$ & $0.46 \mathrm{~m}$ & $2.33 \mathrm{jk}$ & $2.43 \mathrm{kl}$ & $0.65 \mathrm{o}$ & $0.73 \mathrm{k}$ & $0.37 \mathrm{k}$ & $0.37 \mathrm{i}$ & $31.3 \mathrm{kl}$ & $33.50 \mathrm{fg}$ \\
\hline & Moringa & $1.38 \mathrm{k}$ & $1.48 \mathrm{j}$ & $0.44 \mathrm{gh}$ & $0.46 \mathrm{~m}$ & $2.36 \mathrm{i}$ & $2.43 \mathrm{kl}$ & $0.66 \mathrm{n}$ & $0.75 \mathrm{j}$ & $0.38 \mathrm{jk}$ & $0.38 \mathrm{hi}$ & $31.66 \mathrm{jk}$ & $34.00 \mathrm{f}$ \\
\hline & Chitosan & $1.39 \mathrm{k}$ & $1.49 \mathrm{ij}$ & $0.45 \mathrm{fg}$ & 0.471 & $2.37 \mathrm{hi}$ & $2.44 \mathrm{jk}$ & $0.68 \mathrm{~m}$ & $0.75 \mathrm{j}$ & $0.39 \mathrm{ij}$ & $0.39 \mathrm{~h}$ & $32.16 \mathrm{ij}$ & $35.66 \mathrm{e}$ \\
\hline \multirow{4}{*}{$\begin{array}{l}150 \mathrm{~kg} \\
\mathrm{~S} / \text { fed }\end{array}$} & Control & $1.41 \mathrm{j}$ & $1.50 \mathrm{i}$ & $0.41 \mathrm{i}$ & $0.46 \mathrm{~m}$ & $2.33 \mathrm{jk}$ & $2.45 \mathrm{ij}$ & 0.691 & $0.78 \mathrm{i}$ & $0.39 \mathrm{i}$ & $0.41 \mathrm{~g}$ & $32.33 \mathrm{hi}$ & $32.66 \mathrm{~g}$ \\
\hline & Yeast & $1.43 \mathrm{i}$ & $1.53 \mathrm{~h}$ & $0.43 \mathrm{~h}$ & $0.48 \mathrm{k}$ & $2.34 \mathrm{j}$ & $2.46 \mathrm{i}$ & $0.72 \mathrm{k}$ & $0.79 \mathrm{i}$ & $0.40 \mathrm{~h}$ & $0.41 \mathrm{fg}$ & $32.83 \mathrm{~h}$ & $33.83 \mathrm{f}$ \\
\hline & Moringa & $1.44 \mathrm{~h}$ & $1.54 \mathrm{gh}$ & $0.45 \mathrm{fg}$ & $0.49 \mathrm{j}$ & $2.37 \mathrm{gh}$ & $2.48 \mathrm{~h}$ & $0.74 \mathrm{j}$ & $0.81 \mathrm{~h}$ & $0.42 \mathrm{gh}$ & $0.41 \mathrm{f}$ & $33.50 \mathrm{~g}$ & $35.66 \mathrm{e}$ \\
\hline & Chitosan & $1.46 \mathrm{~g}$ & $1.55 \mathrm{fg}$ & $0.47 \mathrm{de}$ & $0.50 \mathrm{i}$ & $2.38 \mathrm{gh}$ & $2.50 \mathrm{~g}$ & $0.75 \mathrm{i}$ & $0.83 \mathrm{~g}$ & $0.43 \mathrm{fg}$ & $0.43 \mathrm{e}$ & $34.16 \mathrm{ef}$ & $36.50 \mathrm{e}$ \\
\hline \multirow{4}{*}{$\begin{array}{l}300 \mathrm{~kg} \\
\mathrm{~S} / \mathrm{fed}\end{array}$} & Control & $1.48 \mathrm{f}$ & $1.54 \mathrm{gh}$ & $0.45 \mathrm{fg}$ & $0.51 \mathrm{~h}$ & $2.38 \mathrm{gh}$ & $2.51 \mathrm{~g}$ & $0.78 \mathrm{~h}$ & $0.85 \mathrm{f}$ & $0.43 \mathrm{fg}$ & $0.44 \mathrm{de}$ & $34.00 \mathrm{fg}$ & $34.00 \mathrm{f}$ \\
\hline & Yeast & $1.49 \mathrm{ef}$ & $1.55 \mathrm{fg}$ & $0.46 \mathrm{ef}$ & $0.52 \mathrm{~g}$ & $2.39 \mathrm{fg}$ & $2.53 \mathrm{f}$ & $0.82 \mathrm{~g}$ & $0.87 \mathrm{e}$ & $0.44 \mathrm{f}$ & $0.44 \mathrm{de}$ & 34.66de & $36.00 \mathrm{e}$ \\
\hline & Moringa & $1.50 \mathrm{de}$ & $1.56 \mathrm{ef}$ & $0.47 \mathrm{de}$ & $0.54 \mathrm{f}$ & $2.40 \mathrm{ef}$ & $2.54 \mathrm{e}$ & $0.83 \mathrm{f}$ & $0.91 d$ & $0.44 \mathrm{e}$ & $0.45 \mathrm{~d}$ & $35.16 \mathrm{~d}$ & $38.00 \mathrm{~d}$ \\
\hline & Chitosan & $1.51 \mathrm{~d}$ & $1.57 \mathrm{de}$ & $0.48 \mathrm{~cd}$ & $0.55 \mathrm{e}$ & $2.41 \mathrm{e}$ & $2.56 \mathrm{~d}$ & $0.85 \mathrm{e}$ & $0.93 \mathrm{c}$ & $0.46 \mathrm{de}$ & $0.45 \mathrm{~d}$ & $36.33 \mathrm{c}$ & $38.50 \mathrm{~cd}$ \\
\hline \multirow{4}{*}{$\begin{array}{l}450 \mathrm{~kg} \\
\mathrm{~S} / \text { fed }\end{array}$} & Control & $1.50 \mathrm{~d}$ & $1.58 \mathrm{~cd}$ & $0.49 \mathrm{c}$ & $0.56 \mathrm{~d}$ & $2.44 \mathrm{~d}$ & $2.57 \mathrm{~cd}$ & $0.91 \mathrm{~d}$ & $0.95 \mathrm{c}$ & $0.47 \mathrm{~d}$ & $0.46 \mathrm{~d}$ & $36.33 \mathrm{c}$ & $38.66 \mathrm{~cd}$ \\
\hline & Yeast & $1.53 \mathrm{c}$ & $1.59 \mathrm{bc}$ & $0.53 \mathrm{~b}$ & $0.58 \mathrm{c}$ & $2.46 \mathrm{c}$ & $2.58 \mathrm{c}$ & $0.93 \mathrm{c}$ & $0.97 \mathrm{~b}$ & $0.46 \mathrm{c}$ & $0.47 \mathrm{bc}$ & $37.16 \mathrm{~b}$ & $39.33 \mathrm{bc}$ \\
\hline & Moringa & $1.55 \mathrm{~b}$ & $1.60 \mathrm{~b}$ & $0.54 \mathrm{~b}$ & $0.60 \mathrm{~b}$ & $2.49 \mathrm{~b}$ & $2.60 \mathrm{~b}$ & $0.94 \mathrm{~b}$ & $0.98 b$ & $0.48 \mathrm{~b}$ & $0.49 \mathrm{~b}$ & $37.66 \mathrm{ab}$ & $40.33 \mathrm{ab}$ \\
\hline & Chitosan & $1.57 \mathrm{a}$ & $1.61 \mathrm{a}$ & $0.58 \mathrm{a}$ & $0.61 \mathrm{a}$ & $2.52 \mathrm{a}$ & $2.61 \mathrm{a}$ & $0.95 \mathrm{a}$ & $1.10 \mathrm{a}$ & $0.51 \mathrm{a}$ & $0.53 \mathrm{a}$ & $38.16 \mathrm{a}$ & $41.00 \mathrm{a}$ \\
\hline
\end{tabular}

Means followed by the same letter in column are not significantly differed according to Duncan's Multiple Range Test at $5 \%$ level of probability. NSS=Natural stimulant substances

Effect of foliar spray with some natural stimulant substances:

Data in Table (5) show that nitrogen, phosphorus, potassium, sulphur, volatile oils and TSS \% contents in garlic cloves at harvest were significantly increased due to spray the plants with chitosan compared with the other natural stimulant treatments. These results agree with those reported by Fawzy et al. (2012), Ahmed (2015) and Hegazi et al. (2016).

Effect of interaction between S-levels and foliar spray with some natural stimulant substances:

It is evident from data in Table (5) that the interaction between the two studied factors i.e.sulphur fertilizer levels and foliar spray with some natural stimulant substances had a significant effects on all chemical constituents in cloves in both seasons. The highest values of nitrogen, phosphorus, potassium, sulphur, volatile oils and TSS \% contents in garlic cloves at harvest were shown when garlic plants supplied $450 \mathrm{~kg} \mathrm{~S} /$ fed and sprayed with chitosan. Similar results were obtained by Ahmed (2015) who found that sulphur application with foliar spray with natural stimulants significantly increased the chemical contents in cloves.

\section{4- Storability:}

Effect of sulphur levels:

Data in Table (6) reveal that the total weight loss percentage of bulbs was significantly affected during storage period in both seasons. The lowest total weight loss percentage was obtained by applied-S at $450 \mathrm{~kg} / \mathrm{fed}$. This treatment achieved increase in yield at the end of storage period (five months) reached to $20.3 \%$ and $20.5 \%$ in the first and the second seasons respectively comparing to control treatment. These results may be due to increase dry matter in plants (Table 2), TSS \% and chemical constituents in cloves (Table 4). These results are in the same line with those of ElMorsy (2005) and Shete et al. (2018).

Effect of foliar spray with some natural stimulant substances:

Data in Table (6) indicate that bulb storability of plants sprayed with some natural stimulant substances was better than that of the untreated plants. Beside, foliar spray with chitosan was more beneficial than the other treatments. These decrease in weight loss percentage in garlic bulb as a result of foliar spraywith some natural stimulant substances may be due to that chitosan is known for its ability to extend the storage life of vegetables, where chitosan forms a semi-permeable film that regulates gas exchange, reduces respiration and transpiration rates and slows down the ripening processes (Shehata et al., 2012). In addition, helpful roles of moringa extract and yeast extract in improving growth, yield attributes and dry matter accumulation in bulbs more than water, therefore improved storability of bulbs during storage periods. The aforementioned results generally are in good agreement with those stated by Shalaby and El-Ramady (2014) and Ahmed (2015). 
Table 6. Weight loss percentage of garlic as affected by sulphur levels, natural stimulant substances and their interactions during 2015P/2016 (S1) and 2016/2017 (S2) seasons.

\begin{tabular}{|c|c|c|c|c|c|c|c|c|c|c|c|}
\hline \multirow{2}{*}{\multicolumn{2}{|c|}{$\begin{array}{l}\text { Characters } \\
\text { Treatments }\end{array}$}} & \multicolumn{2}{|c|}{30 Days } & \multicolumn{2}{|c|}{60 Days } & \multicolumn{2}{|c|}{90 Days } & \multicolumn{2}{|c|}{120 Days } & \multicolumn{2}{|c|}{150 Days } \\
\hline & & S1 & S2 & S1 & S2 & S1 & S2 & S1 & S2 & S1 & S2 \\
\hline \multicolumn{12}{|c|}{ Sulphur levels: } \\
\hline \multicolumn{2}{|l|}{ Control } & $30.97 \mathrm{a}$ & $30.35 \mathrm{a}$ & $41.90 \mathrm{a}$ & $41.35 \mathrm{a}$ & $47.32 \mathrm{a}$ & $45.68 \mathrm{a}$ & $51.32 \mathrm{a}$ & $50.77 \mathrm{a}$ & $55.92 \mathrm{a}$ & $54.80 \mathrm{a}$ \\
\hline \multicolumn{2}{|c|}{$150 \mathrm{~kg} \mathrm{~S} / \mathrm{fed}$} & $26.82 \mathrm{~b}$ & $25.97 \mathrm{~b}$ & $37.97 \mathrm{~b}$ & $38.05 \mathrm{~b}$ & $41.45 \mathrm{~b}$ & $42.29 \mathrm{~b}$ & $47.45 \mathrm{~b}$ & $46.89 \mathrm{~b}$ & $51.55 \mathrm{~b}$ & $50.72 b$ \\
\hline \multicolumn{2}{|c|}{$300 \mathrm{~kg} \mathrm{~S} / \mathrm{fed}$} & $23.70 \mathrm{c}$ & $23.52 \mathrm{c}$ & $34.10 \mathrm{c}$ & $33.52 \mathrm{c}$ & $40.12 \mathrm{~b}$ & $40.42 \mathrm{c}$ & $44.00 \mathrm{c}$ & $44.22 \mathrm{c}$ & $47.21 \mathrm{c}$ & $45.92 \mathrm{c}$ \\
\hline \multicolumn{2}{|c|}{$450 \mathrm{~kg} \mathrm{~S} / \mathrm{fed}$} & $22.30 \mathrm{c}$ & $23.05 \mathrm{~d}$ & $32.45 \mathrm{~d}$ & $31.77 \mathrm{~d}$ & $37.82 \mathrm{~b}$ & $38.70 \mathrm{~d}$ & $40.92 \mathrm{~d}$ & $40.17 \mathrm{~d}$ & $44.20 \mathrm{~d}$ & $42.52 \mathrm{~d}$ \\
\hline \multicolumn{12}{|c|}{ Natural stimulant substances: } \\
\hline \multicolumn{2}{|c|}{ Control } & $29.04 \mathrm{a}$ & $28.50 \mathrm{a}$ & $40.02 \mathrm{a}$ & $40.00 \mathrm{a}$ & $45.97 \mathrm{a}$ & $47.10 \mathrm{a}$ & $50.22 \mathrm{a}$ & $49.60 \mathrm{a}$ & $54.77 \mathrm{a}$ & $53.92 \mathrm{a}$ \\
\hline \multicolumn{2}{|c|}{ Yeast $(100 \mathrm{ml} / \mathrm{L})$} & $26.26 \mathrm{~b}$ & $25.57 \mathrm{~b}$ & $37.12 \mathrm{~b}$ & $36.60 \mathrm{~b}$ & $42.90 \mathrm{~b}$ & $43.22 \mathrm{~b}$ & $46.77 \mathrm{~b}$ & $46.17 \mathrm{~b}$ & $50.82 \mathrm{~b}$ & $49.13 b$ \\
\hline \multicolumn{2}{|c|}{ Moringa $(100 \mathrm{ml} / \mathrm{L})$} & $23.75 \mathrm{~b}$ & $24.87 \mathrm{c}$ & $35.25 \mathrm{c}$ & $34.70 \mathrm{c}$ & $40.90 \mathrm{c}$ & $39.55 \mathrm{c}$ & $44.40 \mathrm{c}$ & $43.89 \mathrm{c}$ & $47.64 \mathrm{c}$ & $46.80 \mathrm{c}$ \\
\hline \multicolumn{2}{|c|}{ Chitosan $(200 \mathrm{mg} / \mathrm{L})$} & $24.74 \mathrm{~b}$ & $23.95 \mathrm{~d}$ & $34.03 \mathrm{~d}$ & $33.40 \mathrm{~d}$ & $36.95 \mathrm{~d}$ & $37.22 \mathrm{~d}$ & $42.40 \mathrm{~d}$ & $42.40 \mathrm{~d}$ & $45.65 \mathrm{~d}$ & $44.12 \mathrm{~d}$ \\
\hline \multicolumn{12}{|c|}{ Interactions: } \\
\hline \multicolumn{12}{|c|}{ S-levels $\quad X \quad$ NSS } \\
\hline \multirow{4}{*}{ Control } & Control & $33.06 \mathrm{a}$ & $32.60 \mathrm{a}$ & $44.60 \mathrm{a}$ & $43.90 \mathrm{a}$ & $51.30 \mathrm{a}$ & $51.80 \mathrm{a}$ & $56.00 \mathrm{a}$ & $55.30 \mathrm{a}$ & $61.80 \mathrm{a}$ & $61.00 \mathrm{a}$ \\
\hline & Yeast & $31.50 \mathrm{ab}$ & $30.80 \mathrm{~b}$ & $42.90 \mathrm{~b}$ & $42.60 \mathrm{~b}$ & $48 \mathrm{ab}$ & $47.40 \mathrm{~b}$ & $52.80 \mathrm{~b}$ & $52.20 \mathrm{~b}$ & $57.30 \mathrm{a}$ & $65.53 b$ \\
\hline & Moringa & $30.00 \mathrm{a}-\mathrm{c}$ & $29.50 \mathrm{c}$ & $41.40 \mathrm{~d}$ & $40.90 \mathrm{c}$ & $46.10 \mathrm{a}-\mathrm{c}$ & $45.70 \mathrm{c}$ & $49.00 \mathrm{~d}$ & $48.50 \mathrm{~d}$ & $53.30 \mathrm{~d}$ & $52.50 \mathrm{c}$ \\
\hline & Chitosan & $29.33 a-c$ & $28.50 \mathrm{~d}$ & $38.73 \mathrm{e}$ & $38.00 \mathrm{~d}$ & $43.90 \mathrm{~b}-\mathrm{d}$ & $43.50 \mathrm{~d}$ & $47.50 \mathrm{f}$ & $47.10 \mathrm{e}$ & $51.10 \mathrm{~g}$ & 49.20de \\
\hline \multirow{4}{*}{$\begin{array}{l}150 \mathrm{~kg} \\
\mathrm{~S} / \text { fed }\end{array}$} & Control & $30.00 \mathrm{a}-\mathrm{c}$ & $29.50 \mathrm{c}$ & $42.20 \mathrm{c}$ & $43.80 \mathrm{a}$ & $47.60 \mathrm{a}-\mathrm{c}$ & $47.13 \mathrm{~b}$ & $52.00 \mathrm{c}$ & $51.30 \mathrm{c}$ & $56.70 \mathrm{c}$ & $55.90 \mathrm{~b}$ \\
\hline & Yeast & $26.16 \mathrm{~cd}$ & $25.30 \mathrm{fg}$ & $37.40 \mathrm{f}$ & $37.00 \mathrm{e}$ & $44 \mathrm{bcd}$ & $43.46 \mathrm{~d}$ & $47.80 \mathrm{ef}$ & $47.20 \mathrm{e}$ & $53.00 \mathrm{e}$ & $52.10 \mathrm{c}$ \\
\hline & Moringa & $25.80 \mathrm{~cd}$ & $24.90 \mathrm{~g}$ & $36.30 \mathrm{j}$ & $35.90 \mathrm{f}$ & 41.90b-e & $41.40 \mathrm{e}$ & $45.00 \mathrm{~h}$ & $44.96 \mathrm{f}$ & $48.50 \mathrm{i}$ & $47.70 \mathrm{e}$ \\
\hline & Chitosan & $25.33 \mathrm{~cd}$ & $24.20 \mathrm{~h}$ & $36.00 \mathrm{gh}$ & $35.50 \mathrm{fg}$ & $32.30 \mathrm{f}$ & $40.90 \mathrm{ef}$ & $45.00 \mathrm{~h}$ & $44.10 \mathrm{~g}$ & $48.00 \mathrm{j}$ & $47.20 \mathrm{ef}$ \\
\hline \multirow{4}{*}{$\begin{array}{l}300 \mathrm{~kg} \\
\mathrm{~S} / \mathrm{fed}\end{array}$} & Control & $26.60 \mathrm{~b}-\mathrm{d}$ & $26.30 \mathrm{e}$ & $37.40 \mathrm{f}$ & $37.00 \mathrm{e}$ & $43.80 \mathrm{~b}-\mathrm{d}$ & $43.10 \mathrm{~d}$ & $48.00 \mathrm{e}$ & $47.40 \mathrm{e}$ & $52.00 \mathrm{f}$ & $51.20 \mathrm{~cd}$ \\
\hline & Yeast & $23.70 \mathrm{~d}$ & $23.00 \mathrm{i}$ & $35.00 \mathrm{i}$ & $34.30 \mathrm{~h}$ & $41.60 \mathrm{~b}-\mathrm{e}$ & $41.00 \mathrm{ef}$ & $45.80 \mathrm{~g}$ & $45.30 \mathrm{f}$ & $49.00 \mathrm{~h}$ & $47.80 \mathrm{e}$ \\
\hline & Moringa & $21.70 \mathrm{~d}$ & $22.80 \mathrm{ij}$ & $32.60 \mathrm{k}$ & $32.00 \mathrm{i}$ & 39.20de & $38.80 \mathrm{~g}$ & $43.70 \mathrm{i}$ & $43.10 \mathrm{~h}$ & $45.86 \mathrm{k}$ & $45.10 \mathrm{f}$ \\
\hline & Chitosan & $22.60 \mathrm{~d}$ & $22.00 \mathrm{k}$ & 31.401 & $30.80 \mathrm{j}$ & $35.90 \mathrm{ef}$ & $35.30 \mathrm{ij}$ & 38.501 & $41.10 \mathrm{i}$ & $42.00 \mathrm{n}$ & $39.60 \mathrm{~h}$ \\
\hline \multirow{4}{*}{$\begin{array}{l}450 \mathrm{~kg} \\
\mathrm{~S} / \mathrm{fed}\end{array}$} & Control & $26.50 b-d$ & $25.60 \mathrm{f}$ & $35.90 \mathrm{~h}$ & $35.30 \mathrm{~g}$ & $41.20 \mathrm{c}-\mathrm{e}$ & $40.70 \mathrm{f}$ & $44.90 \mathrm{~h}$ & $44.40 \mathrm{~g}$ & $48.60 \mathrm{i}$ & $47.60 \mathrm{e}$ \\
\hline & Yeast & $23.70 \mathrm{~d}$ & $23.20 \mathrm{i}$ & $33.20 \mathrm{~g}$ & $32.50 \mathrm{i}$ & $38 \mathrm{def}$ & $37.30 \mathrm{~h}$ & $40.70 \mathrm{j}$ & $40.00 \mathrm{j}$ & 44.001 & 40.10gh \\
\hline & Moringa & $22.90 \mathrm{~d}$ & $22.30 \mathrm{jk}$ & $30.70 \mathrm{~m}$ & $30.00 \mathrm{k}$ & $36.40 \mathrm{ef}$ & $35.80 \mathrm{i}$ & $39.90 \mathrm{k}$ & $38.00 \mathrm{k}$ & $42.70 \mathrm{~m}$ & $41.90 \mathrm{~g}$ \\
\hline & Chitosan & $16.30 \mathrm{e}$ & 21.101 & $30.00 \mathrm{n}$ & 29.301 & $35.70 \mathrm{ef}$ & $35.10 \mathrm{j}$ & 38.201 & 37.301 & $41.50 \mathrm{o}$ & $40.50 \mathrm{gh}$ \\
\hline
\end{tabular}

Means followed by the same letter in column are not significantly differed according to Duncan's Multiple Range Test at $5 \%$ level of probability. NSS=Natural stimulant substances

Effect of interaction between S-levels and foliar spray with some natural stimulant substances:

It is clear from data in Table (6) that the positive interactions between sulphur fertilizer levels and foliar spray with some natural stimulant substances often observed on storability of bulbs. The lowest total weight loss percentage during and in at the end of the storage period (five months) was obtained from plants received $450 \mathrm{~kg} \mathrm{~S} /$ fed and sprayed with chitosan in both seasons.

\section{CONCLUSION}

As results of this study, it could be concluded that, application of $450 \mathrm{~kg} \mathrm{~S} / \mathrm{fed}$ with spray garlic plants with chitosan could be recommended for increasing garlic yield, improving bulb quality and storability of bulbs under similar conditions of this work.

\section{REFERENCES}

A.O.A.C. (1970). Official methods of analysis agricultural chemists, 11th ed. Published by the A.O.A.C., P.O. Boxi : 504 Washington.

Ahmed, M.E.M. (2015). Response of garlic plants (Allium sativum L.) to foliar application of some biostimulants. Egypt. J. Hort., 42(1): 613- 625.

Assefa, A.G. ; S.H. Mesgina and Y.W. Abrha (2015). Effect of inorganic and organic fertilizers on the growth and yield of garlic crop (Allium sativum L.) in Northern Ethiopia. J. Agric. Sci., 7(4): 80-86.
Babaleshwar, S.B. ; R. Shilpa ; K.K. Koppad and R. Dharmatti (2017). Influence of sulphur on growth and yield of garlic (Allium sativum L.). J. of Pharma.andPhytochem., 6(5): 450-452.

Black, C. A. (1965). "Methods of soil analysis".Part 1. Physical andmineralogical.ASA Madison, Wise., USA.

Culver, M. ; T. Fanuel and A.Z. Chiteka (2012). Effect of moringa extract on growth and yield of tomato. Greener J. of Agric. Sci., 2(5): 207-211.

Dahdouh, S.M. ; N.N. Youssef and A.S. Metwally (1993). Sulphur and chicken manure interactions in relation to yield and availability of macro and micronutrients to tomato grown on calcareous soil. Egypt. J. Appl. Sci., 8: 638-653.

Duncan, D.B. (1955). Multiple range and multiple F test. Biometrics, 11: 1-42.

El-Morsy, A.H.A. (2005).Effect of sulpher levels and foliar application of certain micronutrients on garlic (Allium sativum L.). The 6th Arabian Conf. for Horticulture, Ismailia, Egypt, pp: 454-464.

El-Morsy, A.H.A ; U.M. Saif El-Deen and A.S. Ezzat (2011). Response of growth, productivity and storability of garlic (Allium sativum L.) to foliar spray with magnesium and yeast extract J. Plant Production, Mansoura Univ., 2(1): 39-51. 
Farooqui, M.A. ; I.S. Naruka ; S.S. Rathore ; P.P. Singh and R.P.S. Shaktawa (2009). Effect of nitrogen and sulphur levels on growth and yield of garlic (Allium sativum L.). Asian J. of Food and AgroInd., Special Issue, pp: 18-23.

Fawzy, Z.F. ; Z.S. El-Shal ; L. Yunsheng ; O. Zhu and Omaima M. Sawan (2012). Response of garlic (Allium Sativum L.) plants to spraying of some biostimulants under sandy soil condition. J. App. Sci. Res., 8(2): 770-776.

Gomez, K.N. and A.A. Gomez (1984).Statistical procedures for agricultural research. John Wiley and Sons, New York, 2nd ed., $68 \mathrm{p}$.

Gunther, E, (1961). "The Essential Oils" 4th . Ed. Vol. 1 D., van Nostrand Co. Inc., New York.

Hegazi, Amal Z. ;Shimaa, Kh.H. Hasan and Nadia, A.M. El-Said (2016). Response of garlic plants to foliar application of moringa leaves extract, glutamine and cysteine. J. Plant Production, Mansoura Univ., 7(1): $1-6$.

Hesse, P.R. (1971). A text book of Soil Chemical Analysis. John. Murray (Publish), London, Great Britain.

Hore, J.K. ; S. Ghanti and M. Chanchan (2014). Influence of nitrogen and sulphur nutrition on growth and yield of garlic (Allium sativum L.). J. of Crop and Weed, 10(2):14-18.

Hunter, A.H. (1984). Soil Analytical Services in Bangladesh. BARI/Aids Consultancy Report. Contract Aid/388-005, Dhaka. Bangladesh. Pp: 1-7.

Jackson, M.L. (1967). Soil Chemical Analysis.Pintic Hall of India, pp. 144-147.

Jaggi, R.C. and S.K. Raina (2005). Nutrient content in garlic (Allium sativum) as indices of yield and yield attributes. Agropedology, 15, 107-109.

Mann, L.K. (1952). Anatomy of the garlic bulb and factors affecting bulb development. Hilgardia, 21: 195-228.

Marschner, H. (2012). Mineral nutrition of higher plants.Academic press San Diego, USA.
Mehana, T.A. (1994). Microbiological properties and nutrient availability in a salt-affected calcareous soil as influenced by certain amendments under leaching conditions. Bull. Suez Canal Univ. Appl. Sci., 3: 384-402.

Mehana, T.A. and F.M. Farag (2000). Influence of phosphate-dissolving micro-organisms and elemental sulphur on phosphorus and micronutrient availability in a calcareous soil treated with rock phosphate. J. Agric. Sci. Mansoura Univ., 25 (5): 2983-2993.

Patidar, M. ; R.P.S. Shaktawat and I.S. Naruka (2017). Effect of sulphur and vermin-compost on growth, yield and quality of garlic (Allium sativum L.). J. KrishiVigyan, 5(2): 54-56.

Shalaby, T.A. and H. El-Ramady (2014).Effect of foliar application of bio-stimulants on growth, yield, components, and storability of garlic (Allium sativum L.). Australian J. of Crop Sci., 8(2): 271275.

Shehata, S.A. ; Z.F. Fawzy and H.R. El-Ramady (2012). Response of cucumber plants to foliar application of chitosan and yeast under greenhouse conditions. Australian J. of Basic and App. Sci., 6(4): 63-71.

Shete, M.B. ; H.M. Chiktey ; S.B. Jadhav and M.N. Bhalekar (2018). Effect of sulphur on growth, yield and quality of garlic (Allium sativum L.).Intern. J. Chem. Studies, 6(1): 552-555.

Spencer, T.F.T. ; S.M. Dorothy and A.R.W. Smith (1983). Yeast genetics "fundamental and applied aspects", pp: 16-18, ISBN 387-390973-9, Springer. Verlag. New York, U.S.A.

Youssif, B.D. ; S. Hosna ; A.F. Mahmoud and Mervat A.T. Amara (2015). Effect of sulphur and sulphur oxidizing bacteria on growth and production of garlic (Allium sativum, L.) under saline conditions. Middle East J. Agric. Res., 4(3): 446-459.

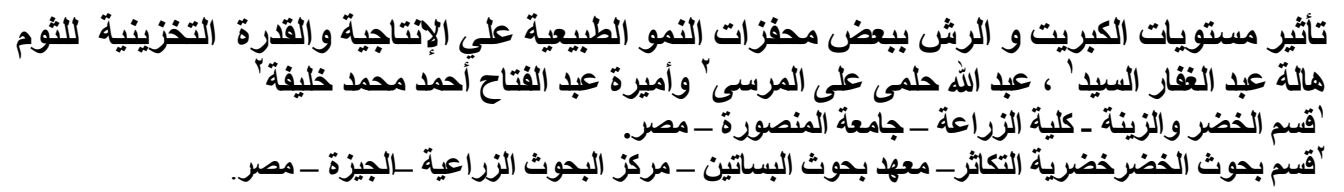

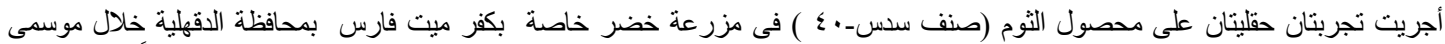

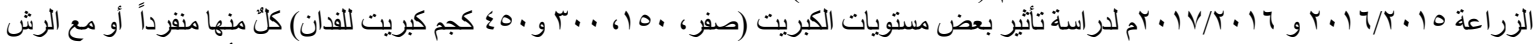

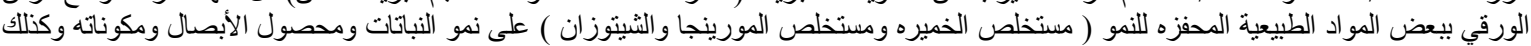

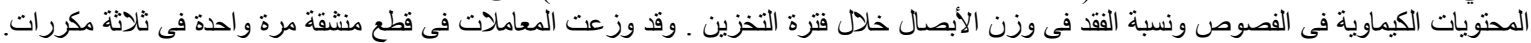

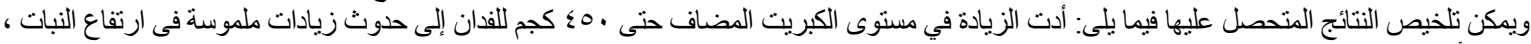

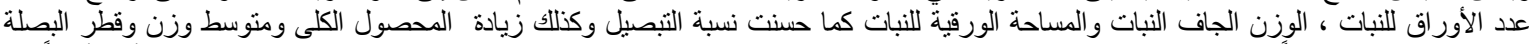

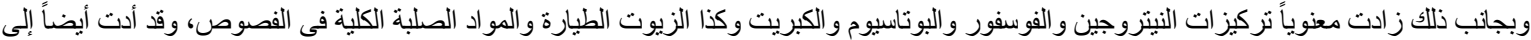

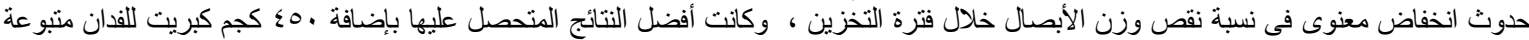

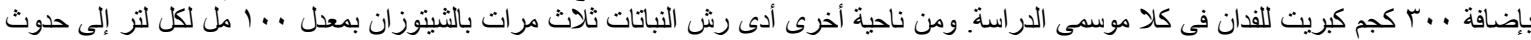

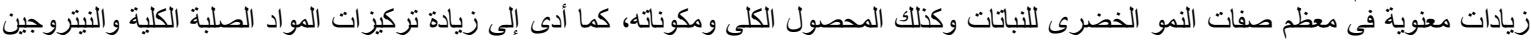

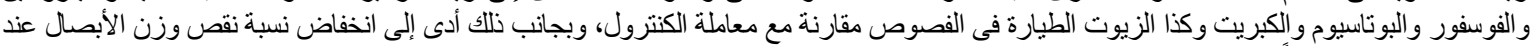

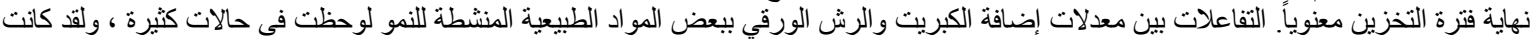

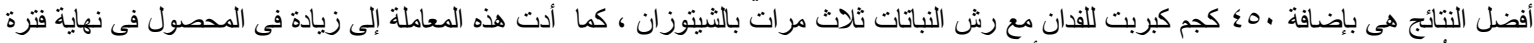

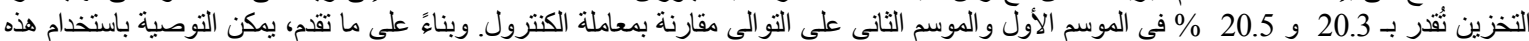

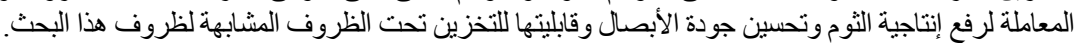

